\title{
INTEGRATION OF DESIGN AND ANALYSIS THROUGH BOUNDARY INTEGRAL EQUATIONS
}

\author{
Benjamin Marussig $^{1}$, Jürgen Zechner ${ }^{1}$, Gernot Beer ${ }^{1,2}$, and Thomas-Peter Fries ${ }^{1}$ \\ ${ }^{1}$ Institute of Structural Analysis \\ Graz University of Technology \\ Lessingstraße 25/II, 8010 Graz, Austria \\ e-mail: \{marussig,juergen.zechner,fries\}@ tugraz.at \\ ${ }^{2}$ Centre for Geotechnical and Materials Modeling \\ University of Newcastle Callaghan, NSW 2308, Australia \\ e-mail: gernot.beer@tugraz.at
}

Keywords: Isogeometric Analysis, Boundary Element Method, Trimmed NURBS, Independent Field Approximation, Extended B-splines.

\begin{abstract}
The direct integration of Computer Aided Geometric Design (CAGD) models into a numerical simulation improves the accuracy of the geometrical representation of the problem as well as the efficiency of the overall analysis process.

In this work, the complementary features of isogeometric analysis and boundary integral equations are combined to obtain a coalescence of design and analysis which is based on a boundary-only discretization. Following the isogeometric concept, the functions used by CAGD are employed for the simulation. An independent field approximation is applied to obtain a more flexible and efficient formulation. In addition, a procedure is presented which allows a stable analysis of trimmed geometries and a straightforward positioning of collocation points.

Several numerical examples demonstrate the characteristics and benefits of the proposed approach. In particular, the independent field approximation improves the computational efficiency and reduces the storage requirements without any loss of accuracy. The proposed methodology permits a seamless integration of the most common design models into an analysis of linear elasticity problems.
\end{abstract}




\section{INTRODUCTION}

Isogeometric analysis aims to close the existing gap between the design process and analysis such that a simulation can be performed without generating a mesh. Consequently, the accuracy and efficiency of the overall simulation process is improved, since meshing is timeconsuming [1,2] and introduces additional (geometrical) approximation errors. In addition, the basis functions used by design models, i.e. NURBS, provide further benefits such as high continuity $[3,4]$.

However, during the last years, it has become clear that a true integration of design and analysis is far from trivial due to several reasons: first of all, most engineering design models are based on a boundary representation (B-Rep) rather than a volume description. Secondly, three dimensional B-Rep models are usually defined by a non-conforming partition of NURBS surfaces, i.e. their mathematical parametrizations have no explicit relation to each other. Thirdly, each boundary surface is based on a tensor product structure which is a very efficient representation but has limitations due to its four sided nature. As a result, almost all NURBS based design models use trimming procedures to increase the flexibility of tensor product surfaces. This means that only a certain area of a surface is visualized while the underlying mathematical parametrization remains unchanged.

In this work, a coherent framework is presented which allows a seamless integration of trimmed NURBS models into an analysis. In general, the governing equations of the problem are expressed by means of boundary integral equations which are discretized by a numerical approximation method. Here, the boundary element method (BEM) is used since it is the most versatile approach. However, it should be pointed out that other schemes like the Nyström method can be applied as well $[5,6]$. An independent field approximation concept is introduced in order to obtain a flexible BEM formulation. Furthermore, this allows the stabilization of trimmed NURBS geometries by the application of extended B-splines. The following sections provide an overview of the proposed methodology. They actually recap and unify the main features presented in [6-8]. Hence, the interested reader is particularly referred to the first one of these references for an in-depth discussion.

\section{METHODOLOGY}

\subsection{Isogeometric Boundary Element Method}

A linear elastic body $\Omega$ subject to external loading without body forces is considered. The closed boundary of the domain is denoted by $\Gamma$ and the surface normal $\mathbf{n}$ points outside. Using Betti's or Green's theorem the following integral equation also known as Somigliana's identity is obtained

$$
\mathbf{u}(\boldsymbol{x})=\int_{\Gamma} \mathrm{U}(\boldsymbol{x}, \boldsymbol{y}) \mathbf{t}(\boldsymbol{y}) \mathrm{ds}_{\boldsymbol{y}}-\int_{\Gamma} \mathrm{T}(\boldsymbol{x}, \boldsymbol{y}) \mathbf{u}(\boldsymbol{y}) \mathrm{ds}_{\boldsymbol{y}} \quad \forall \boldsymbol{x} \in \Omega, \forall \boldsymbol{y} \in \Gamma
$$

where $\mathrm{U}$ and $\mathrm{T}$ are fundamental solutions for displacement and traction, respectively [9]. In general, a fundamental solution $\mathrm{U}(\boldsymbol{x}, \boldsymbol{y})$ provides the response at a field point $\boldsymbol{y}$ due to a unit point source applied at $\boldsymbol{x}$, which is denoted as source point. It should be noted that once the Cauchy data, i.e. displacement $\mathbf{u}(\boldsymbol{y})$ and traction $\mathbf{t}(\boldsymbol{y})$, are known on the entire boundary $\Gamma$, the representation formula (1) describes the displacement $\mathbf{u}(\boldsymbol{x})$ within the whole domain $\Omega$. In order to solve for unknown boundary values, the source points $\boldsymbol{x}$ are shifted to $\Gamma$ leading to the 
boundary integral equation

$$
\mathbf{c}(\boldsymbol{x}) \mathbf{u}(\boldsymbol{x})=\int_{\Gamma} \mathrm{U}(\boldsymbol{x}, \boldsymbol{y}) \mathbf{t}(\boldsymbol{y}) \mathrm{ds}_{\boldsymbol{y}}-\int_{\Gamma} \mathrm{T}(\boldsymbol{x}, \boldsymbol{y}) \mathbf{u}(\boldsymbol{y}) \mathrm{ds}_{\boldsymbol{y}} \quad \forall \boldsymbol{x}, \boldsymbol{y} \in \Gamma
$$

in which the coefficient $\mathbf{c}(\boldsymbol{x})$ depends on the geometrical angle of $\Gamma$ at $\boldsymbol{x}$ and the Poisson's ratio [10]. Equation (2) represents the governing equations of the problem by means of an integral over the boundary of the computational domain. Hence, corresponding numerical approximation methods like the BEM do not require a domain discretization.

In isogeometric boundary element formulations, the geometry as well as the boundary data are represented by B-spline or NURBS basis functions. The boundary $\Gamma$ of the computational domain is specified by a disjoint set of patches (curves or surfaces) $\gamma$ such that

$$
\Gamma=\bigcup_{i=1}^{I} \gamma_{i}
$$

which is equivalent to the representation of design models. Since this is the best geometry representation available the computational boundary is described as accurate as possible.

Within each $\gamma$, the geometry $\boldsymbol{x}(\xi)$ is defined by a set of basis functions $B_{i, p}$ of degree $p$ with corresponding coefficients in physical space $\boldsymbol{c}_{i}$ which are denoted as control points. The geometrical mapping $\chi$ from parameter space $\xi$ to physical space is given by

$$
\chi(\xi):=\boldsymbol{x}(\xi)=\sum_{i=0}^{I-1} B_{i, p}(\xi) \boldsymbol{c}_{i}
$$

with $I$ representing the total number of basis functions. The main advantages of these patches are that their continuity is directly controlled by the applied basis functions. The parameter space $\xi$ as well as the properties of this basis functions are determined by a knot vector $\Xi$ which is a non-decreasing sequence of parametric coordinates $\xi_{i}$. For more detailed information on B-splines and NURBS the interested reader is referred to [2, 11]. Similar to the geometric mapping (4), displacements and tractions of each $\gamma$ are discretized by

$$
\mathcal{Y}_{u}(\xi):=\mathbf{u}(\xi)=\sum_{i=0}^{I-1} B_{i}(\xi) \tilde{\mathbf{u}}_{i} \quad \text { and } \quad \mathcal{Y}_{t}(\xi):=\mathbf{t}(\xi)=\sum_{j=0}^{J-1} B_{j}(\xi) \tilde{\mathbf{t}}_{j} .
$$

The coefficients $\tilde{\mathbf{u}}_{i}$ and $\tilde{\mathbf{t}}_{j}$ are control parameters of the corresponding field and the related basis functions are denoted by $B_{i}$ and $B_{j}$.

The system of equations is set up using a collocation approach where the boundary integral equation is enforced in a set of collocation points $\boldsymbol{x}^{c}$. In particular, each basis function $B_{i}$ of the unknown field is related to a certain $\boldsymbol{x}_{i}^{c}$. It has been demonstrated by several authors $[7,12,13]$ that the Greville abscissae

$$
\bar{\xi}_{i}=\frac{\xi_{i+1}+\xi_{i+2}+\cdots+\xi_{i+p}}{p}
$$

are a robust and accurate choice for the location of the collocation points, i.e. $\boldsymbol{x}_{i}^{c}=\chi\left(\bar{\xi}_{i}\right)$. For each $\boldsymbol{x}_{i}^{c}$, the integrals of the boundary integral equation (2) with $\boldsymbol{x}=\boldsymbol{x}_{i}^{c}$ are evaluated using numerical integration. Since each $\gamma$ may contain non-smooth regions, it has to be subdivided 
into integration elements. Within those integration elements, the integrand is smooth and hence numerical integration can be performed properly.

Usually, equation (6) leads to some collocation points located on the boundary of each $\gamma$. Hence, they should be considered during the integration of adjacent $\gamma$ as well. For two dimensional problems, i.e. boundary curves, this is not challenging. However, in case of boundary surfaces, detection of adjacent surfaces and determination of the collocation points' intrinsic coordinates within them may be very involved. Especially, if non-conforming partitions of trimmed surfaces are considered. The application of discontinuous collocation is an elegant remedy to this issue $[6,7]$. Using such schemes, collocation points along the boundary of $\gamma$ are slightly shifted inside, thereby abolishing the link to adjacent surfaces. In general, this is possible since interelement continuity is not necessarily required in BEM [14].

\subsection{Independent Field Approximation}

In the context of isogeometric BEM, the Cauchy data are discretized by means of B-splines or NURBS. The related mappings (5) are denoted by $\mathcal{Y}_{u}(\xi)$ and $\mathcal{Y}_{t}(\xi)$ for displacements $\mathbf{u}$ and traction $\mathbf{t}$, respectively. In the following, the distinction between isoparametric and subparametric patches is introduced. The former employs the isoparametric paradigm, i.e. all fields are represented by the same basis functions, as it is generally the case in isogeometric analysis. The latter utilizes the proposed independent field approximation. The term subparametric indicates that less parameters are used for the description of the geometry than for the Cauchy data.

\subsubsection{Isoparametric Patches}

In an isoparametric discretization, the mappings $\mathcal{Y}_{u}(\xi)$ and $\mathcal{Y}_{t}(\xi)$ are equal to the geometrical one $\chi(\xi)$. This implies some compromises. First of all, the same refinement is applied to all fields. On the one hand, refinement of the unknown field is mandatory to improve the solution. But as a consequence, the geometry and the known field are refined even though they may be exactly represented by the initial basis functions. In addition to refinement aspects, the fields have different continuity requirements along corners and edges. In particular, discontinuous basis functions are required to describe traction jumps. However, they are not optimal for representing the displacement field, which should be continuous according to the physical constraint. Furthermore, the number of basis functions would be increasing unnecessarily.

Thus, a preliminary conclusion is that an isoparametric discretization introduces superfluous control variables. This increases the numerical effort and storage requirements for setting up the system of equations, particularly for its right hand side. Moreover, refinement of CAGD models affects the efficiency of all geometry evaluations performed during the analysis. These points motivate the application of the proposed subparametric approach presented next.

\subsubsection{Subparametric Patches}

In this section, subparametric patches are introduced. The key idea is to treat each field separately in order to fulfill their individual needs. In particular, the concept of a subparametric element is adopted, i.e. more basis functions will be used to represent the field variables than for the geometry representation. The basis functions of the geometry, the displacements, and the tractions are defined by the knot vector $\Xi_{g}, \Xi_{u}$, and $\Xi_{t}$, respectively. Without loss of generality, basis functions of the Cauchy data are defined as an extended version of $\Xi_{g}$, so that $\Xi_{g} \subset \Xi_{u}$ and $\Xi_{g} \subset \Xi_{t}$. This definition guarantees a proper partition of integration elements. It should, 
however, be noted that it allows the variation of basis functions types. For instance, the Cauchy data over a NURBS patch may be approximated by B-splines. The resulting benefits are that the evaluation of Cauchy data becomes more efficient and the refinement procedure is simplified. Moreover, it has been demonstrated that the approximation quality is hardly effected by such a combination $[7,13]$.

In the present implementation the geometry knot vector $\Xi_{g}$ provides the initial basis for all fields. Subsequently, basis functions are refined only if it is necessary. Discontinuous basis functions are introduced only for the traction field at non-smooth boundaries. The known Cauchy data are classified as simple or complex boundary conditions as indicated in Figure 1. The former can be exactly represented by $\Xi_{g}$, hence the corresponding discretization does not need to be refined. Homogeneous boundary conditions or constant loading are examples for such boundary conditions. The latter can only be approximated and the corresponding basis functions have to be refined to improve the representation of the known Cauchy data. In such a case, refinement is performed equally to the one for the approximation of the unknown field.

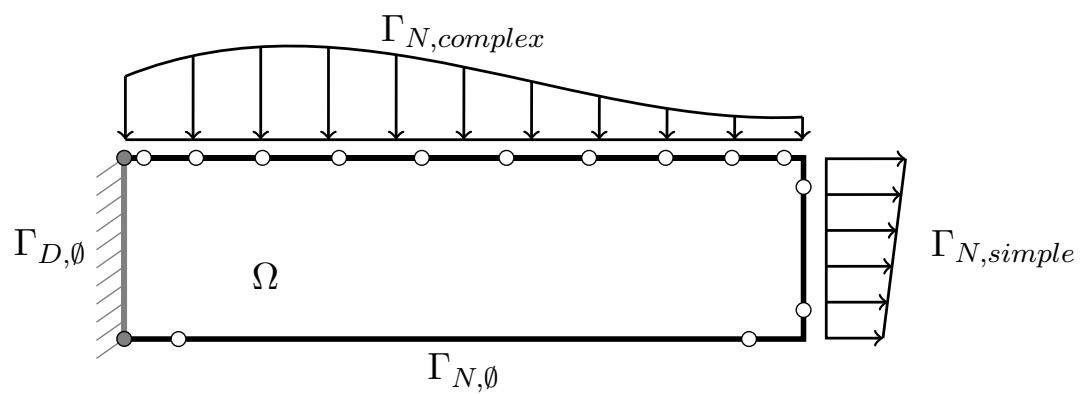

Figure 1: Examples of simple and complex boundary conditions applied to a cantilever beam. Homogeneous boundary condition are denoted by the subscript $\emptyset$. Circles indicate the related Greville abscissae of the basis functions which represent the known data.

To sum up, the main aim of independent field approximation is to avoid redundancies in the discretization, leading to an enhanced isogeometric BEM formulation. At the same time, no discretization errors are introduced and the initial design model remains unchanged during the simulation which simplifies the interaction with CAGD software.

\subsection{Stable Basis for Trimmed Geometries}

In general, a trimmed parameter space has only a certain area which represents the part of an object that is visualized. This part is denoted as valid domain $\Omega^{\mathrm{v}}$ for the remainder of this paper. Usually, trimming is used for surfaces and in this case $\Omega^{\mathrm{v}}$ is determined by so-called trimming curves which are defined within the parameter space of the surface.

There are two main aspects that have to be considered if trimmed geometries are integrated into a numerical simulation: firstly, only the visualized part has to be considered for the analysis. Secondly, the trimmed parameter space has to be stabilized, because basis functions with small support occur which may lead to ill-conditioned system matrices. Several different approaches [15-20] have been proposed to address the former issue. However, the latter has hardly been considered in the literature so far. Hence, the current section focuses on this aspect. In particular, it is proposed to stabilize trimmed parameter spaces by using so-called extended $B$-splines which have been originally introduced in the context of fictitious domain - finite element methods [21-24]. 


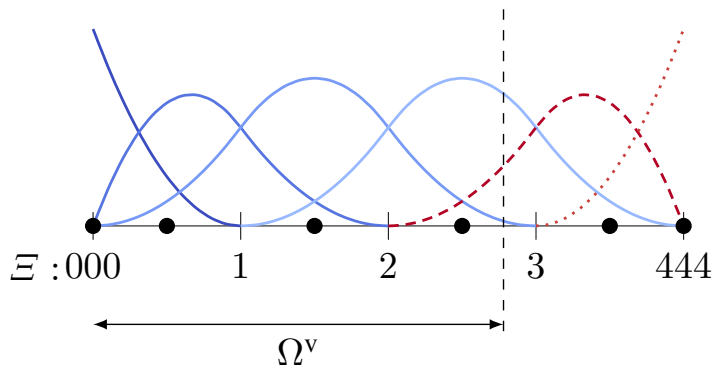

Figure 2: Classification of basis function types in case of a trimmed parameter space: stable (continuous), degenerated (dashed), and exterior (dotted). The related Greville abscissae are illustrated by circles.

The basic idea of extended B-splines is to replace basis functions which may cause instabilities by extrapolations of neighboring ones which have a sufficient large support. In order to classify the B-splines of a trimmed parameter space, three types are introduced: stable, degenerated, and exterior. We propose to label B-splines $B_{i, p}$ as stable if their corresponding Greville abscissae is within the valid domain, i.e. $\bar{\xi}_{i} \in \Omega^{\mathrm{v}}$. The support of exterior B-splines, on the other hand, is entirely outside of $\Omega^{\mathrm{v}}$. The remaining degenerated basis functions are partially inside of $\Omega^{\mathrm{v}}$ but their Greville abscissae is outside, i.e. $\bar{\xi}_{i} \notin \Omega^{\mathrm{v}}$. These various types are shown for an univariate parameter space in Figure 2. Once the degenerated B-splines are detected, the functions within their support are substituted by extensions of the closest stable segments as illustrated in Figure 3. The resulting extended B-splines $B_{i, p}^{e}$ are defined by a linear combination of the original ones

$$
B_{i, p}^{e}=B_{i, p}+\sum_{j \in \mathbb{J}_{i}} e_{i, j} B_{j, p}
$$

where $\mathbb{J}_{i}$ is an index-set of all degenerated B-splines $B_{j, p}$ related to the current $B_{i, p}^{e}$. For univariate basis functions, the corresponding extrapolation weights $e_{i, j}$ are generally defined by the so-called de Boor-Fix or dual functional (see e.g. [6, 23, 25]). In addition, a simplified formula can be derived for uniform parameter spaces $[22,24]$. In the bivariate case, the extrapolation weights are simply determined by the tensor product of univariate values.

Extended B-splines inherit most properties of conventional B-splines and provide a stable basis for the analysis of trimmed geometries. The proposed strategy for detecting degenerated basis functions is particularly beneficial for collocation schemes, because the definition of the collocation point locations within $\Omega^{\mathrm{v}}$ is straightforward, i.e. equal to the non-trimmed situation.

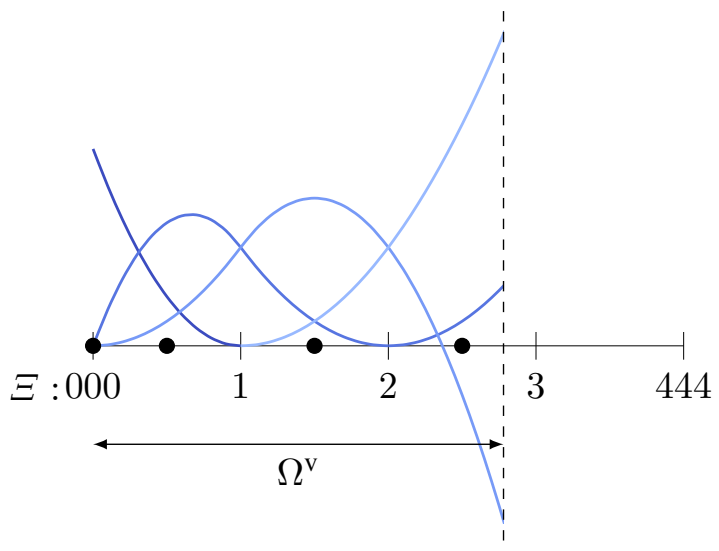

Figure 3: Extended B-splines 
The presented concept focuses on B-spline basis functions. However, if the geometry is defined by trimmed NURBS, we take advantage of the independent field approximation and simply approximate the Cauchy data by B-splines without modifying the geometrical representation.

\section{NUMERICAL RESULTS}

The following examples emphasize the benefits of the proposed methodology. Firstly, the advantage of accurate geometry models is investigated. Secondly, the performance of isogeometric and subparametric patches is compared. Finally, the approximation quality of extended B-splines is studied.

\subsection{Sphere}

The excavation of a spherical cavity with radius $r_{s}=5.0 \mathrm{~m}$ is investigated. An isotropic elastic material is considered with Poisson's ratio $\nu=0.3$ and Young's modulus $E=1000 \mathrm{MPa}$. Hydrostatic stress $\sigma_{0}=1.0 \mathrm{MPa}$ is applied as loading which leads to a uniform internal pressure along the excavation surface. The resulting radial displacement $u_{r}$ can be determined analytically by

$$
u_{r}=\sigma_{0} \frac{1+\nu}{2 E} \frac{r_{s}^{3}}{r^{2}}
$$

where $r$ denotes the distance of the point observed to the center of the sphere. Hence, the reference solution for $u_{r}$ along the boundary, i.e. $r=r_{s}$, is $3.25 \times 10^{-3} \mathrm{~m}$.

The problem is solved by conventional and isogeometric BEM simulations. Both approaches employ quadratic basis functions. The conventional BEM meshes approximate the boundary of the computation domain with quadratic Serendipity elements. In the isogeometric case, NURBS basis function of degree $p=2$ are used. In fact, the geometry can be represented exactly by a single NURBS patch which has degenerated edges at each pole of the sphere. This model has been exported from the CAGD software Rhinoceros, where the precision of the exported data was set to $\epsilon_{e}=10^{-8}$. Discontinuous collocation is applied in order to deal with the degenerated edges of the model.

The relative error of the radial displacement $\epsilon_{u_{r}}$ as well as the relative deviation of the geometry representation to an analytical sphere $\epsilon_{g e o}$ are summarized for various degrees of freedom $n$ in Table 1. Further, three numerical results and their corresponding discretizations are illustrated in Figure 5 and Figure 4, respectively.

\begin{tabular}{cccccc}
\hline & \multicolumn{2}{c}{ Isogeometric BEM } & \multicolumn{3}{c}{ Conventional BEM } \\
$n$ & $\epsilon_{u_{r}}$ & $\epsilon_{\text {geo }}$ & $n$ & $\epsilon_{u_{r}}$ & $\epsilon_{\text {geo }}$ \\
\hline 216 & $1.74 \times 10^{-5}$ & $3.42 \times 10^{-8}$ & 483 & $1.61 \times 10^{-2}$ & $4.76 \times 10^{-1}$ \\
288 & $2.65 \times 10^{-6}$ & $3.42 \times 10^{-8}$ & 2436 & $1.27 \times 10^{-3}$ & $1.53 \times 10^{-2}$ \\
& & & 9312 & $5.58 \times 10^{-4}$ & $1.81 \times 10^{-3}$ \\
& & & 12120 & $4.42 \times 10^{-4}$ & $1.10 \times 10^{-3}$ \\
& & & 21858 & $1.49 \times 10^{-4}$ & $3.21 \times 10^{-4}$ \\
& & & 38886 & $7.52 \times 10^{-5}$ & $1.08 \times 10^{-4}$ \\
\hline
\end{tabular}

Table 1: Relative error of solution $\epsilon_{u_{r}}$ and geometry representation $\epsilon_{g e o}$ of the spherical excavation measured in $L_{2}$-norm due to an isogeometric and conventional BEM analysis with quadratic basis functions. 


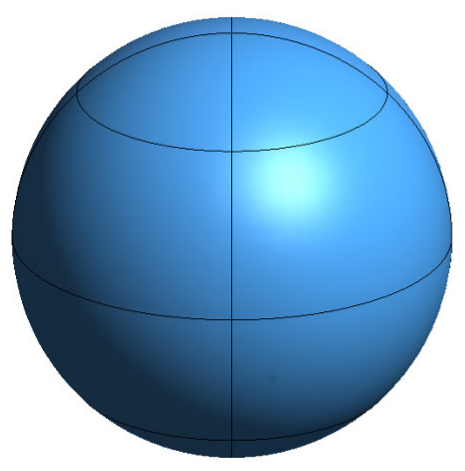

(a) $n=288$

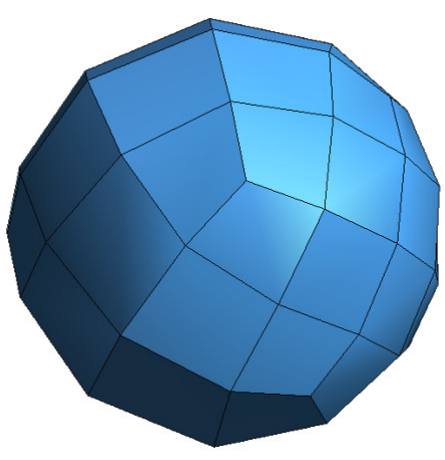

(b) $n=483$

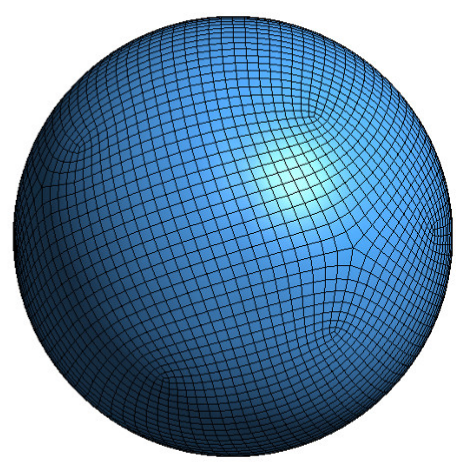

(c) $n=38886$

Figure 4: Various unknown field discretizations of the spherical cavity with different degrees of freedom $n$ : (a) finest isogeometric BEM, (b) coarsest, and (c) finest conventional BEM analysis.

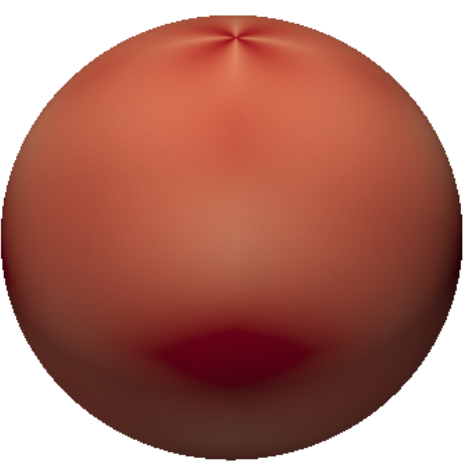

(a) $n=288$

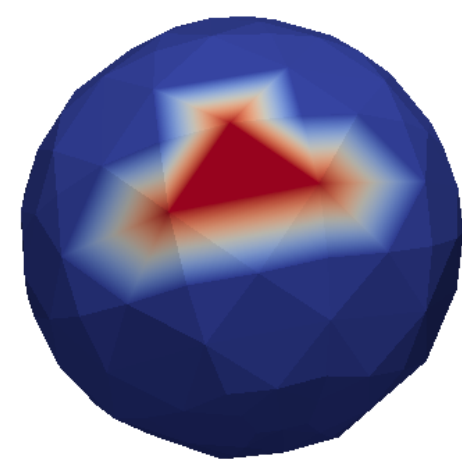

(b) $n=483$

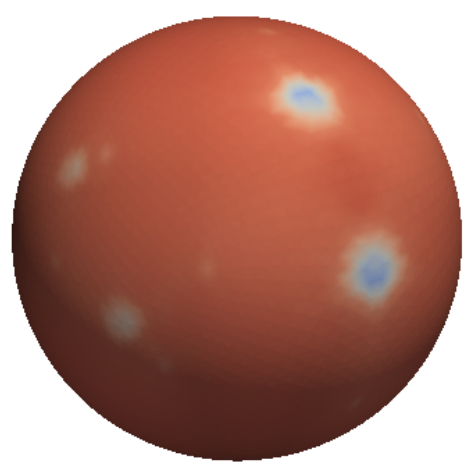

(c) $n=38886$

$3.24943 \times 10^{-3}$

$3.25003 \times 10^{-3}$

Figure 5: Radial displacement $u_{r}$ of various spherical excavation discretizations: (a) finest isogeometric BEM, (b) coarsest, and (c) finest conventional BEM analysis.

The error $\epsilon_{g e o}$ demonstrates clearly the superiority of the isogeometric concept concerning accurate geometry representations. The unrefined NURBS patch provides already a precise geometric model, while a large number of Serendipity elements is required for an adequate approximation. Note that $\epsilon_{g e o}$ correlates to the accuracy of the input data $\epsilon_{e}$ in the isogeometric case, hence it can be controlled by the user. Moreover, the isogeometric solution provides excellent results for $u_{r}$ despite of the low number of degrees of freedom $n$. It should, however, be noted that the example suits NURBS basis functions ideally. Such remarkable differences between isogeometric and conventional BEM solutions can not be expected in general. Nevertheless, conic sections and their three dimensional counterparts, e.g. spheres and cylinders, are very common design elements for which NURBS surpass the approximation quality of conventional basis functions.

\subsection{Crankshaft}

The concept of independent field approximation is applied to a crankshaft example. The geometry is defined by several regular NURBS patches and illustrated in Figure 6. Displacements are fixed at the axle and flywheel, while the crank pins are subjected to vertical loading. These boundary conditions are exactly represented by the initial discretization and do not require re- 


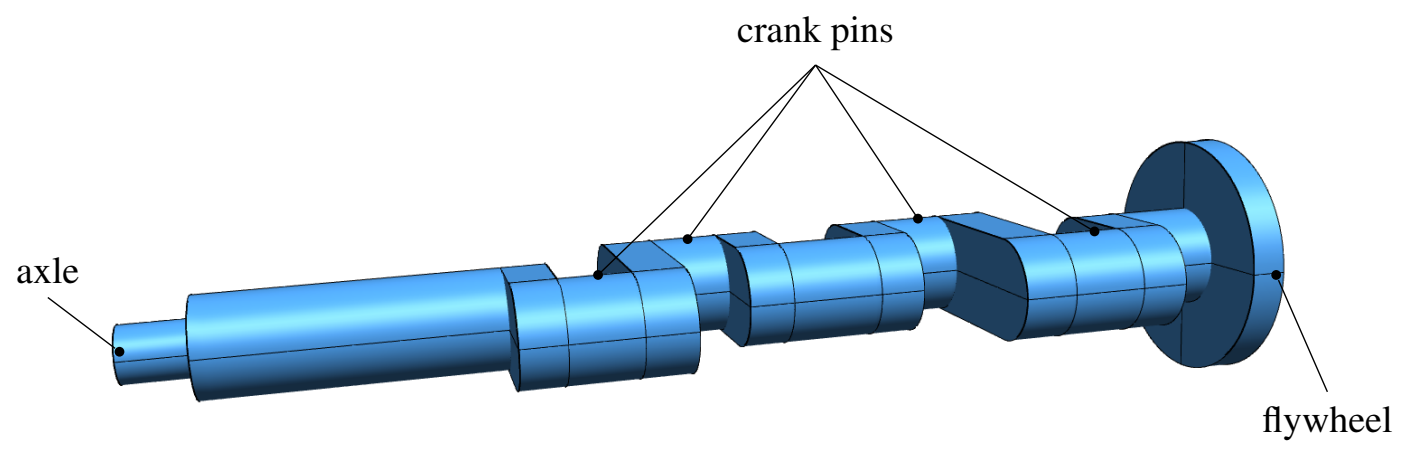

Figure 6: Geometry model of the crankshaft.

finement. The material property is specified by $E=210 \mathrm{GPa}$ and $\nu=0.25$.

Subparametric and isoparametric discretizations are applied to the problem. They differ not only in the refinement procedure, but the type of basis function used for representing the Cauchy data. In particular, B-splines are employed in the subparametric case. The degree of the basis functions related to Cauchy data $p_{c}$ is either equal to the one of the geometry representation $p_{g}$ or increased by means of degree elevation, i.e. $p_{c}=p_{g}+1$. Subsequently, knot insertion is used to improve the results, which is the equivalent to $h$-refinement in conventional analysis. For each simulation, the analysis time $t_{i}$ and $t_{s}$ of the isoparametric and subparametric discretization are compared. To be precise, the runtime for setting up the left hand side matrix $\mathbf{L}$ and the right hand side matrix $\mathbf{R}$ of the system of equations is measured. Each analysis has been performed single-threaded, concurrently for each $t_{i}$ and $t_{s}$, and repeated several times.

The resulting speedup factors $t_{i} / t_{s}$ are summarized in Figure 7 . In addition, the displacements corresponding to the third $h$-refinement step of the case where $p_{c}=p_{g}$ are exemplarily shown in Figure 8.

$\mathbf{L}$

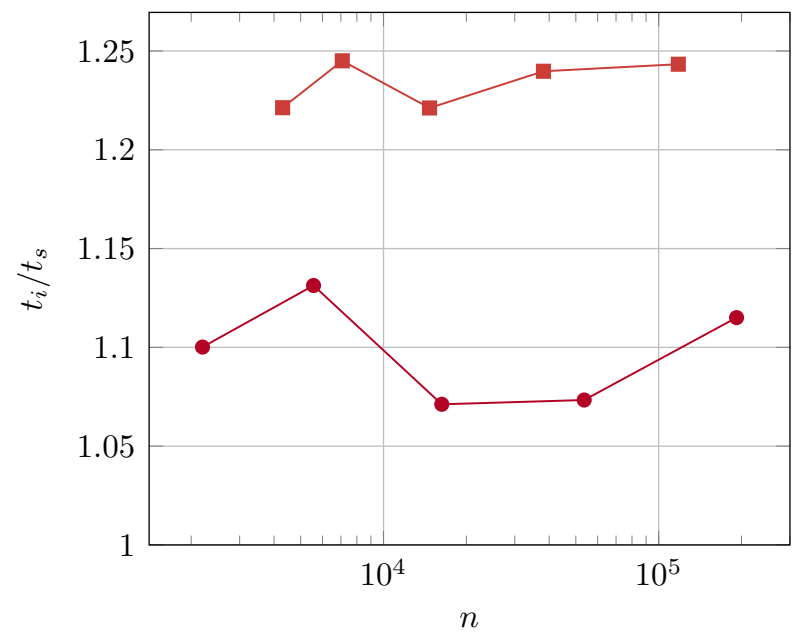

$\mathbf{R}$

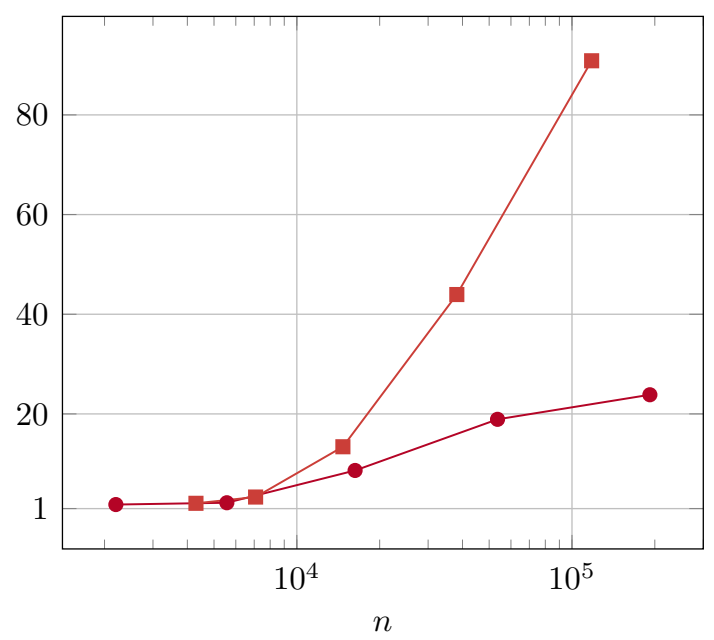

$$
\multimap p_{c}=p_{g} \rightarrow-p_{c}=p_{g}+1
$$

Figure 7: Computational time for the set up of $\mathbf{L}$ and $\mathbf{R}$ related to various discretization of the crankshaft example as a function of the degrees of freedom $n$. The runtime of the isoparametric discretization $t_{i}$ is related to the subparametric one $t_{s}$. 


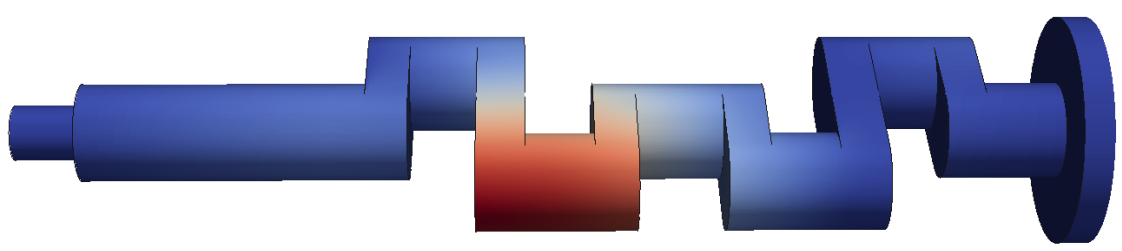

(a) Isoparametric

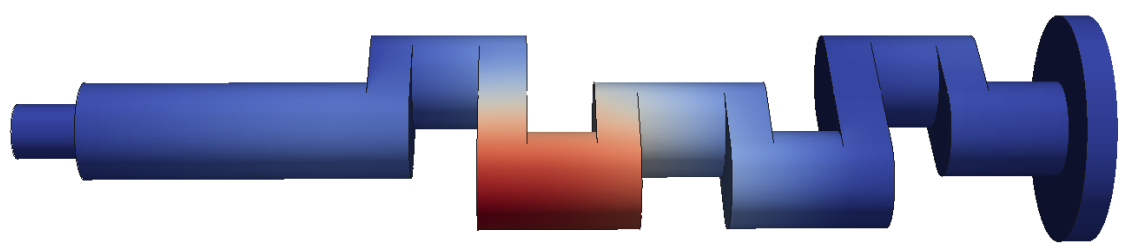

(b) Subparametric

0.000

$1.607 \times 10^{-4}$

Figure 8: The absolute displacement $|\mathbf{u}|$ of the crankshaft example without degree elevation and three $h$-refinement steps due to an (a) isoparametric BEM and (b) subparametric BEM discretization.

The shown results indicate that independent field approximation reduces the computational effort, especially for the right hand side of the system of equations. The number of columns of $\mathbf{R}$ is constant for all subparametric discretizations, because known Cauchy data do not need to be refined. Hence, its storage requirement is linear with respect to the degrees of freedom $n$ which is the driving force for the enormous speedup with respect to $\mathbf{R}$. The efficient geometry evaluation as well as the substitution of NURBS by B-splines are the key factor regarding the faster computation of $\mathbf{L}$ in the subparametric case. The former impact is clearly indicated by the additional offset between the graphs related to $p_{c}=p_{g}$ and $p_{c}=p_{g}+1$ on the left hand side of Figure 7.

\subsection{Trimmed Cube}

In order to investigate the approximation quality of extended B-splines in the context of an isogeometric BEM analysis a unit cube is analyzed. The geometry is discretized by two different models as illustrated in Figure 9. One is described by 6 regular patches, whereas 4 trimmed patches are included in the other. Both represent the same geometry, i.e. $\ell_{x}=\ell_{y}=\ell_{z}=1.0$,

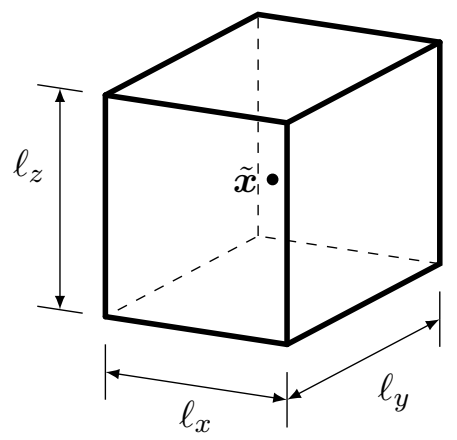

(a) Untrimmed

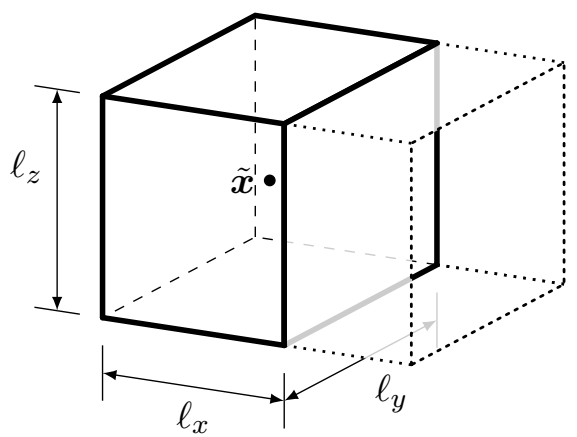

(b) Trimmed

Figure 9: Discretization of a unit cube by (a) regular patches and (b) trimmed patches. 


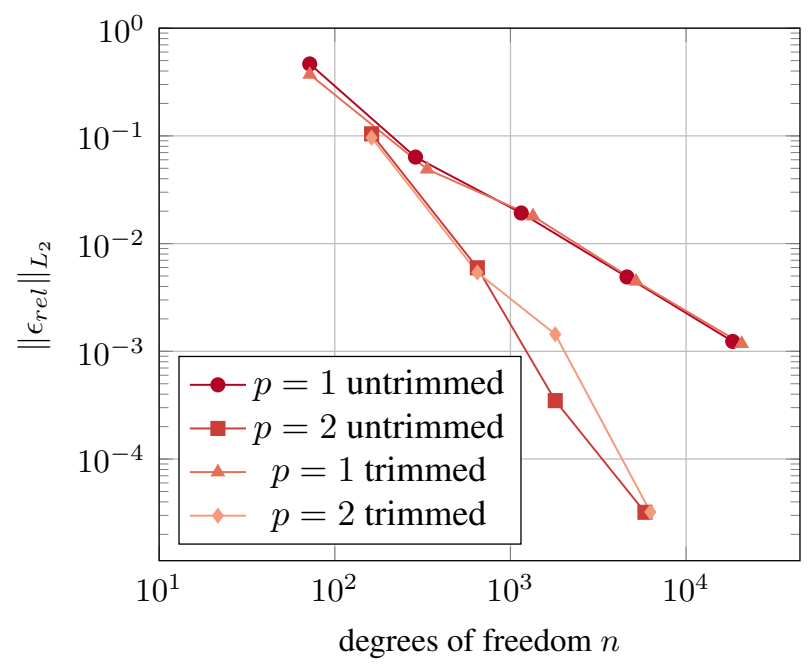

Figure 10: Relative $L_{2}$-error of an exterior Neumann problem on the (trimmed) cube example with respect to the number of degrees of freedom $n$.

which defines the boundary $\Gamma$ of an infinite domain $\Omega$. The boundary condition is given by

$$
\mathbf{t}(\boldsymbol{y})=\mathrm{T}(\tilde{\boldsymbol{x}}, \boldsymbol{y}) \quad \boldsymbol{y} \in \Gamma, \tilde{\boldsymbol{x}} \in \Omega^{-}
$$

with $\Omega^{-}$denoting the void, i.e. $\tilde{\boldsymbol{x}} \notin \Omega$. In particular, a source point $\tilde{\boldsymbol{x}}$ in the center of the cube defines the boundary conditions for the exterior Neumann problem. The discretizations are set up for different degrees $p=\{1,2\}$ and knot insertion is applied to improve the solutions. The relative approximation error is determined by

$$
\epsilon_{r e l}=\frac{\mathbf{u}(\boldsymbol{y})-\mathrm{U}(\tilde{\boldsymbol{x}}, \boldsymbol{y})}{\mathrm{U}(\tilde{\boldsymbol{x}}, \boldsymbol{y})} \quad \forall \boldsymbol{y} \in \Gamma, \tilde{\boldsymbol{x}} \in \Omega^{-}
$$

where $\mathbf{u}(\boldsymbol{y})$ is the obtained solution along the boundary. In Figure 10, the results measured with respect to the $L_{2}$-norm, i.e. $\left\|\epsilon_{\text {rel }}\right\|_{L_{2}}$, are shown. It can be observed that the trimmed model shows very good agreement with the untrimmed case.

\section{CONCLUSIONS}

A comprehensive concept for integrating design models into a numerical simulation has been presented. The essential ingredient is that the isogeometric method applied is based on boundary integral equations. This allows to overcome the challenge of deriving a volume discretization from a design model, in a simple and elegant manner. In addition, no connectivity information is required between adjacent patches. The proposed concept is completed by an independent field approximation paradigm allowing a more flexible interaction with the design model and a technique which stabilizes trimmed parameter space. These enhancements are by no means restricted to the boundary element method used in this work.

The presented results demonstrate the superiority of isogeometric schemes regarding accurate geometry description, the computational advantage of independent field approximation related to conventional isoparametric formulations, and the suitability of the proposed stabilization. The latter results are indeed very promising and deserves to be investigated in more detail. For instance, the behavior for higher degree approximations has been neglected in the present work and is of particular interest for further research. 


\section{REFERENCES}

[1] M. F. Hardwick, R. L. Clay, P. T. Boggs, E. J. Walsh, A. R. Larzelere, A. Altshuler. DART system analysis. Technical Report SAND2005-4647, Sandia National Laboratories, 2005.

[2] J. A. Cottrell, T. J. R. Hughes, Y. Bazilevs, Isogeometric analysis: Toward integration of $C A D$ and FEA. John Wiley \& Sons, Chichester, England, 2009.

[3] J. A. Cottrell, A. Reali, Y. Bazilevs, T. J. R. Hughes, Isogeometric analysis of structural vibrations. Computer Methods in Applied Mechanics and Engineering, 195(41-43), 52575296, 2006.

[4] J. A. Cottrell, T. J. R. Hughes, A. Reali, Studies of refinement and continuity in isogeometric structural analysis. Computer Methods in Applied Mechanics and Engineering, 196(41-44), 4160-4183, 2007.

[5] J. Zechner, B. Marussig, G. Beer, T.-P. Fries, The isogeometric Nyström method. Computer Methods in Applied Mechanics and Engineering, submitted. available as preprint:http://arxiv.org/abs/1506.03914.

[6] B. Marussig. Seamless integration of design and analysis through boundary integral equations. PhD thesis, Graz University of Technology, 2015.

[7] B. Marussig, J. Zechner, G. Beer, T.-P. Fries, Fast isogeometric boundary element method based on independent field approximation. Computer Methods in Applied Mechanics and Engineering, 284, 458-488, 2015.

[8] G. Beer, B. Marussig, C. Duenser, Isogeometric boundary element method for the simulation of underground excavations. Géotechnique Letters, 3, 108-111, 2013.

[9] G. Beer, I. M. Smith, C. Duenser, The boundary element method with programming. Springer Wien - New York, 2008.

[10] V. Mantic, A new formula for the C-matrix in the Somigliana identity. Journal of Elasticity, 33, 191-201, 1993.

[11] L. Piegl, W. Tiller, The NURBS book. Springer, New York, 2 edition, 1997.

[12] F. Auricchio, L. Beirão Da Veiga, T. J. R. Hughes, A. Reali, G. Sangalli, Isogeometric collocation methods. Mathematical Models and Methods in Applied Sciences, 20(11), 2075-2107, 2010.

[13] K. Li, X. Qian, Isogeometric analysis and shape optimization via boundary integral. Computer-Aided Design, 43(11), 1427-1437, 2011.

[14] C. Patterson, M. Sheikh, Interelement continuity in the boundary element method. In C. Brebbia, editor, Topics in Boundary Element Research, 123-141. Springer US, 1984.

[15] G. Beer, B. Marussig, J. Zechner, A simple approach to the numerical simulation with trimmed CAD surfaces. Computer Methods in Applied Mechanics and Engineering, 285, 776-790, 2015. 
[16] H. Harbrecht, M. Randrianarivony, From Computer Aided Design to wavelet BEM. Computing and Visualization in Science, 13(2), 69-82, 2010.

[17] H.-J. Kim, Y.-D. Seo, S.-K. Youn, Isogeometric analysis for trimmed CAD surfaces. Computer Methods in Applied Mechanics and Engineering, 198(37-40), 2982-2995, 2009.

[18] H.-J. Kim, Y.-D. Seo, S.-K. Youn, Isogeometric analysis with trimming technique for problems of arbitrary complex topology. Computer Methods in Applied Mechanics and Engineering, 199(45-48), 2796-2812, 2010.

[19] R. Schmidt, R. Wüchner, K.-U. Bletzinger, Isogeometric analysis of trimmed NURBS geometries. Computer Methods in Applied Mechanics and Engineering, 241-244, 93$111,2012$.

[20] Y.-W. Wang, Z.-D. Huang, Y. Zheng, S.-G. Zhang, Isogeometric analysis for compound B-spline surfaces. Computer Methods in Applied Mechanics and Engineering, 261-262, $1-15,2013$.

[21] K. Höllig, U. Reif, J. Wipper, Weighted extended B-spline approximation of Dirichlet problems. SIAM Journal on Numerical Analysis, 39(2), 442-462, 2002.

[22] K. Höllig, Finite element methods with B-splines, Vol. 26 of Frontiers in Applied Mathematics. SIAM, 2003.

[23] K. Höllig, U. Reif, Nonuniform web-splines. Computer Aided Geometric Design, 20(5), 277-294, 2003.

[24] T. Rüberg, F. Cirak, Subdivision-stabilised immersed b-spline finite elements for moving boundary flows. Computer Methods in Applied Mechanics and Engineering, 209-212(0), 266-283, 2012.

[25] C. de Boor, A practical guide to splines, Vol. 27 of Applied Mathematical Sciences. Springer, New York, 2001. 